\title{
Perivascular Adductor Longus muscle injury: Ultrasound and Magnetic Resonance Imaging findings
}

\author{
Gabriele Mattiussi ${ }^{1}$ \\ Pietro Tobia Baldassi ${ }^{1}$ \\ Giulio Pasta ${ }^{2}$ \\ Aldo Burani ${ }^{3}$ \\ Carlos Moreno ${ }^{1}$ \\ 1 Medical Services, Udinese Football Club, Udine, Italy \\ 2 Imaging Services, Parma Football Club, Parma, Italy \\ 3 Department of Radiodiagnostics, Ospedale di Sas- \\ suolo, Modena, Italy
}

Corresponding author:

Carlos Moreno

Medical Services,

Udinese Football Club

Stadio Friuli, Dacia Arena

Viale A. Candolini 2

33100 Udine, Italy

E-mail: Carlos.Moreno@udinesespa.it

\section{Summary}

Background: Muscle injuries affecting the Adductor Longus are not all localised at the level of the proximal myotendinous junction and enthesis. Thus, the main purpose of this article was to raise awareness of the imaging features of the Perivascular Adductor Longus muscle injury, which currently remains widely under-recognised.

Methods: The ultrasound (US) and Magnetic Resonance imaging (MRI) images of five professional football players were retrospectively reassessed to identify distinctive imaging details of the Perivascular Adductor Longus muscle injury. Complementary information regarding the traumatic mechanics is presented as well.

Results: All the players presented similar US images in the first seventy-two hours: loss of ecostructural integrity of the lateral epimysium, in proximity to the femoral vessels, and perilesional oedema were the main pathological findings. The injury lead to the formation of a hypoechoic, intramuscular haematoma in three of the subjects. Anyway, this was detectable only after five days, or later. Moreover, MRI sequences showed long-standing haematoma-related signal alterations which were also observable at three months after trauma. Typically, kicking was the traumatic motor task.
Conclusions: The main practical value of this technical note is to compensate for the lack of studies concerning the Perivascular Adductor Longus muscle injury. Promptly identifying its typical imaging features is crucial in order to establish the correct diagnosis and to implement a highly specific rehabilitative program.

Level of evidence: $\mathrm{V}$.

KEY WORDS: magnetic resonance imaging, soft tissue injuries, sports injuries, thigh, ultrasonography.

\section{Introduction}

Adductor Longus ( $\mathrm{AL}$ ) muscle injuries, caused by indirect trauma, predominantly involve the proximal myotendinous junction and enthesis ${ }^{1-3}$. These injuries are suspected when the patients refer the acute onset of pain in the pubic or inguinal area, particularly during sporting activities that involve motor tasks such as kicking or twisting movements ${ }^{3-4}$. Hypertrophic scarring (fibrosis) and calcifications are the most common complications of the healing process ${ }^{2}$, as well as a possible cause of long-standing groin pain ${ }^{1,5,6}$. It is worth noting that these abnormalities occur more frequently when both the direct tendon and/or the intramuscular tendon are directly involved, same as for the Quadriceps and Hamstring muscles ${ }^{7}$. However, despite the fact that researchers tend to focus on injuries of the proximal aspects, there are other muscle injury, in other sites, that may affect the AL. Nevertheless, these injuries are still poorly and sparsely documented in literature ${ }^{8-10}$. For this reason, they may remain widely under-recognised and, therefore, inadequately treated. Further studies are needed to raise awareness of the clinical and imaging features of the Perivascular Adductor Longus muscle injury.

Thus, the aims of this article were: I) to present a case series of professional football (soccer) players with a history of $A L$ muscle injury that we have identified as having Perivascular Adductor Longus muscle injury, without any concomitant lesion of the proximal portions of the muscle and II) to define the Ultrasound (US) and Magnetic Resonance Imaging (MRI) features of this peculiar muscle injury, offering practical guidance on how to effectively investigate it. The opinion of the Authors is that the structural abnormalities of the Perivascular Adductor Longus muscle injury are very complicated to detect during US investi- 
gation, as the lesion is localised very close to the femoral vessels (posterior acoustic enhancement artifact-related issue). As regards MRI, instead, the signal alterations can be visualised clearly in just a few sections, in particular, using fat-suppressed fluid sensitive techniques.

\section{Methods}

\section{Participants}

We completed a retrospective reanalysis of a series of eleven footballers, members of the same professional club, who suffered from acute AL muscle injury, caused by indirect trauma, during the three previous sporting seasons (period: July 2013-May 2016). This was done in order to identify individuals who were affected by isolated Perivascular Adductor Longus muscle injury. Five of these athletes were diagnosed with the latter injury and thus included in this research.

This study was conducted in accordance with the ethical standards of the Muscles, Ligaments and Tendons Journal ${ }^{11}$. In addition, it was designed taking into consideration the guidelines for the writing of a technical note ${ }^{12}$.

\section{Review of the cases}

The US and MRI images of the five participants were retrospectively reassessed to identify distinctive imaging details of the Perivascular Adductor Longus muscle injury. The latter mainly involves the lateral peripheral aspects of the muscle, in the middle third level of its belly, or those in direct contact with the Vastus Medialis and femoral blood vessels -hence the "perivascular" prefix- without any concomitant lesion of the proximal portions (myotendinous junction and enthesis) of the muscle.

Complementary information regarding the injury mechanism was noted as well. On the contrary, since the participants of this study were professional football players, basic anthropometric and sport-related details have not been collected in order to ensure maximum anonymization. The US images were captured in the club facilities, using the Logiq S7 Expert (GE Healthcare) device, equipped with a $50 \mathrm{~mm}$ linear probe $(6-15 \mathrm{MHz})$, by an operator with more than ten years of experience in the US assessment of muscle injuries in professional football players. On the other hand, the MRI images were obtained using the HDe 1.5T Signa device (GE Healthcare) in a private clinic. All the US and MRI figures presented in this article have been revised and selected by the Authors for their educational value.

\section{Ultrasound examination: anatomical considera- tions, Push-to-View tip and guidelines}

A thorough knowledge of the US-specific anatomy of the $A L$ is essential to properly complete the investigation. The muscle belly of the AL is easily observable already at 1-2 cm below its insertion on the pubic tubercle, where its short intramuscular tendon is also discernible ${ }^{13,14}$. At this level, between the $A L$ and the adductor canal, also called Hunter's or subsartorial canal, the Pectineus muscle is interposed (Fig. 1) 15-17. By moving the probe distally, almost to the level of the femoral insertion to the Pectineus, the AL comes into direct anatomical contact with the Vastus Medialis and the femoral vessels ${ }^{15}$. Considering the transverse section, the neurovascular bundle is located

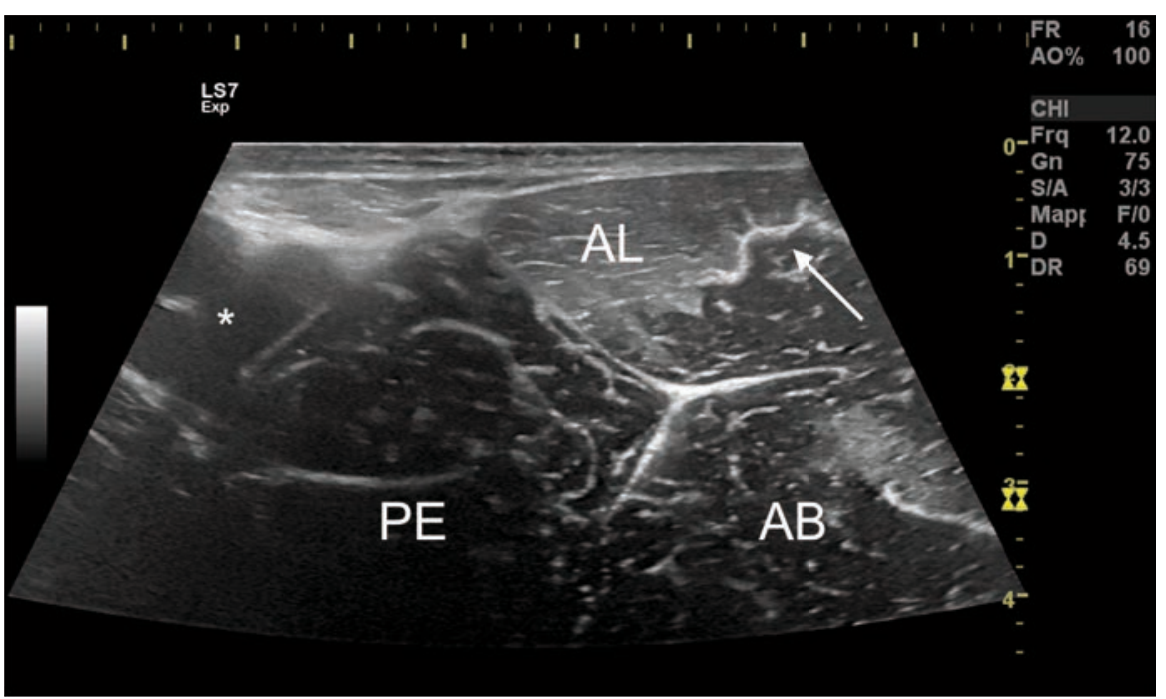

Figure 1. Cross-sectional ultrasound anatomy of the Adductor Longus $(A L)$, proximal third, in a 25-year-old male healthy volunteer. The muscle is easily recognizable as its intramuscular tendon (hyperechoic structure indicated by the arrow) runs through the muscle belly; this has a wavy shape and runs substantially in the coronal plane of AL, almost parallel to the long axis of the muscle. At this level, the AL is still divided from the femoral vessels (asterisk) by the Pectineus muscle (PE). Virtual Convex technology was used to capture this image. $A B=$ Adductor Brevis. 


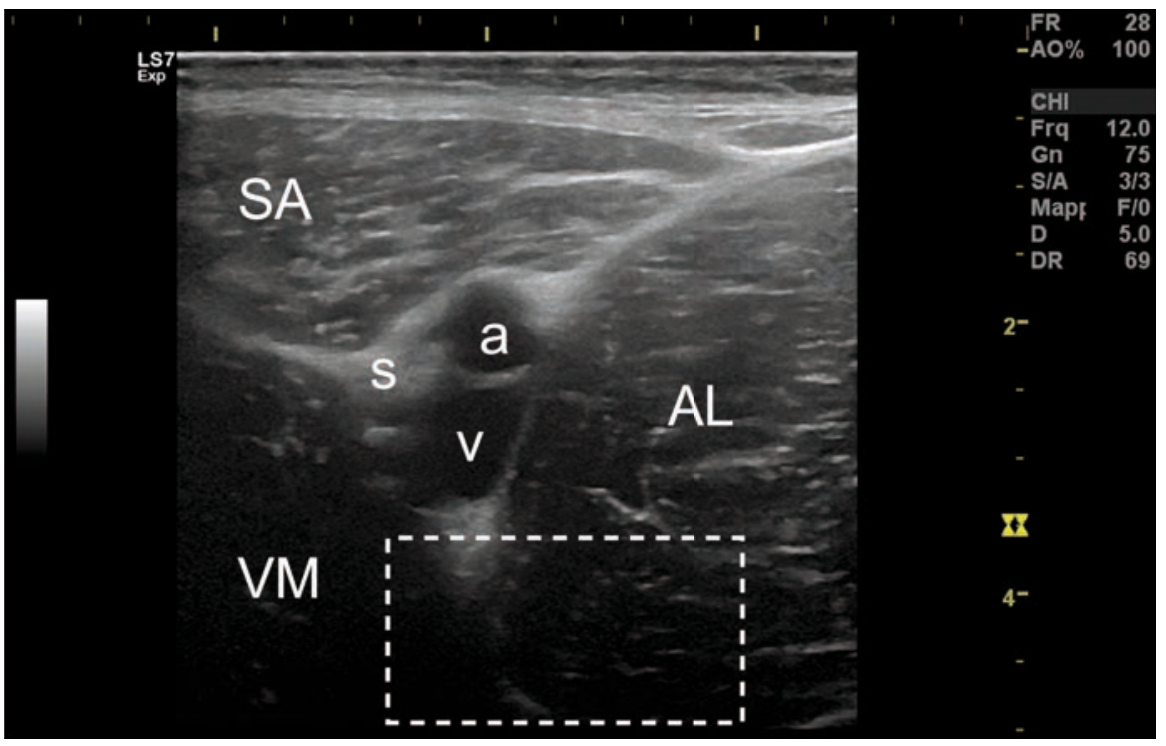

Figure 2. Cross-sectional ultrasound anatomy of the Adductor Longus $(A L)$, middle third, in a 25-year-old male healthy volunteer. The muscle makes anatomical contact with the Vastus Medialis (VM), the femoral vein $(v)$ and the femoral artery $(a)$. Even the saphenous nerve (s), the final branch of the femoral nerve, can be observed. The Sartorious muscle (SA) acts as a "roof" to the neurovascular bundle. It can be seen how the lateral aspects of the AL (inside the dashed rectangle) are not easily observable; this is because the artifact induced by the femoral vein limits their visualisation and due to the fact that they are already at about four centimetres of depth (see parameters of the device on the right).

latero-superiorly to the muscle: usually, the lateral peripheral aspects of the $\mathrm{AL}$ are not simple to investigate due to the posterior acoustic enhancement artifact induced by the blood vessels ${ }^{18}$ and due to the depth at which they are situated (Fig. 2). In addition to this, even the deep femoral vessels and the obturator nerve cannot be seen clearly using high ultrasonic frequencies.

Typically, during the US examination, no pressure must be exerted on the patient's skin with the probe. However, pressure can be applied to compress (or move) a vein. When examining the $A L$, to better visualise the lateral peripheral aspects of the muscle, it is useful to implement the following tip which, for convenience, the Authors named Push-to-View: the posterior acoustic enhancement induced by the femoral vein may be substantially reduced, occluding its blood flow. This can be obtained by applying a moderately high pressure with the probe. In this way, the artifact is reduced sufficiently so as to make the epimysium of the $A L$, the deep femoral vessels and the obturator nerve ideally observable. At this point, the operator is able to adequately observe the site where the Perivascular Adductor Longus muscle injury is located, or the anatomical region interposed between the superficial and the deep femoral vessels (Fig. 3). Assessing also all other portions of the muscle, in particular the proximal myotendinous junctions and enthesis, is mandatory to rule out other injuries.

The ISMuLT guidelines for the US assessment of muscle injuries ${ }^{19}$ and those presented by other Authors ${ }^{20-22}$ have been taken into high consideration during the acquisition and the revision of the US im- ages, in order to be consistent with the most recent and widely used classification systems and with the related nomenclature. All the participants of this study were evaluated every day during the first week post trauma and (at least) twice a week during the following period, up to three months after the traumatic event.

\section{MRI investigation: routine protocol, specific re- quirements and guidelines}

MRI was considered the gold standard to confirm the presence of muscle injuries that were clinically and ultrasonographically suspected ${ }^{19,21-24}$. The protocol included traversal (axial), sagittal and coronal T1weighted spin-echo (T1w), fat-suppressed T2-weighted spin-echo (T2w) and short tau inversion recovery (STIR) sequences ${ }^{20}$. According to the most recent MRI-based classification systems ${ }^{22,23}$, the presence of isolated intramuscular oedema was indicative of a minor lesion, while the presence of perifascial liquid and/or intramuscular haematoma typically indicated a more severe injury. MRI was especially useful to accurately identify the site of injury (myofascial, myotendinous junction, intratendinous or a combination of these) $20,22,23$.

In order to accomplish these aims, transverse MRI sequences were also acquired at the level of the $A L$ muscle belly and not "concentrated" only in the proximal third, as usually done when an Adductor Longus muscle injury is suspected. This was essential to rule out or confirm the presence of Perivascular Adductor Longus muscle injury.

According with the most known and considered 


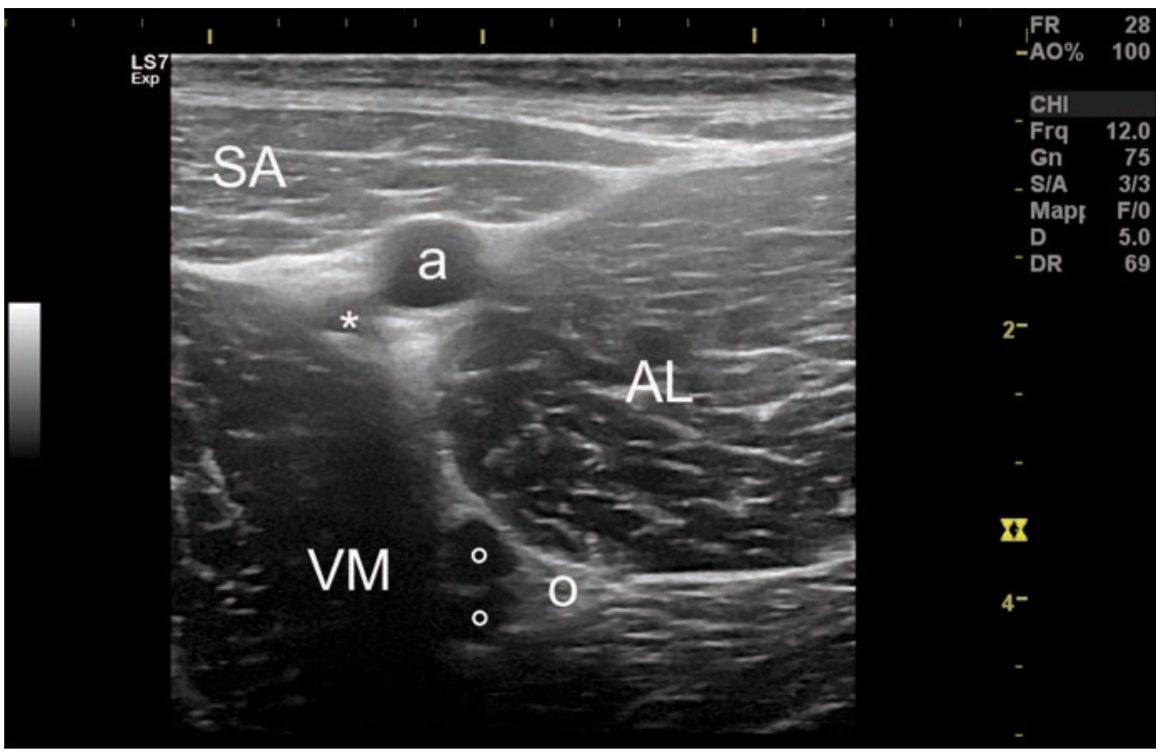

Figure 3. The "Push-to-View" tip, performed in a 25-year-old male healthy volunteer. The ultrasonographer applies pressure to the muscle belly of the Sartorius $(S A)$, using the probe; note the evident flattening of the muscle belly onto the femoral artery (a). In doing so, the blood flow of the femoral vein (asterisk) is almost interrupted, as well as the resulting posterior acoustic enhancement artifact. As a result, the epimysium and perimysium in the lateral portions of the Adductor Longus $(A L)$ become easily observable. In addition to this, thanks to the compression of tissues, even the deep femoral vessels (small circles) and the obturator nerve (large circle) are visible deeper. Therefore, the "perivascular" portion of the AL is now completely observable.

guidelines ${ }^{19}, 20,22,23$, the first MRI was preferably performed within 72 hours after the trauma and, typically, following ultrasound evaluation, while the following two acquisitions were carried out at 3 weeks/1 month and at $2 / 3$ months post-trauma, in all the five footballers.

\section{Results}

\section{Ultrasound}

In the first seventy-two hours, the Perivascular Adductor Longus muscle injury was characterised by: I) loss of eco-structural integrity of the AL lateral epimysium, which appeared hypo-echogenic; II) intramuscular, perilesional oedema, corresponding to the areas of increased echogenicity within the muscle belly and III) difficulty seeing the perilesional perimysium, due to the intramuscular oedema. All the five players presented comparable images in this phase (Fig. 4, Tab. I).

The difference in the image quality captured by applying the Push-to-View tip, or not, was critical for diagnosis in all the cases (Fig. 5). Despite this, those signs were challenging to detect or of controversial analysis and interpretation in two footballers (Fig. 6, Tab. I). It is worth noting that, in this first phase, the US signs were similar even among injuries that have proven to be, afterward, of different severity.

The muscle injury led to the local formation of intramuscular haematoma in three of five cases (Figs. 7, $8)$. This was defined by the presence of hypo- echogenic or anechoic areas within the muscle belly. Interestingly, intramuscular haematoma was not evident before day five following the traumatic event, in all of these three cases (Tab. I).

\section{Magnetic resonance imaging}

In the first seventy-two hours, the Perivascular Adductor Longus muscle injury was characterised by: I) altered signal of the lateral fascial aspects of the AL; II) altered signal of the lateral muscle fibres and III) presence of inter-fascial liquid infiltrations, even detectable distally to the lesion.

Transversal sequences acquired using fat-suppressed fluid sensitive techniques were basically used to assess the signs listed above. In T2w transversal sequences the signal alteration, albeit possibly moderate, mimics a pseudo-thickening of the lateral fascial aspects, making it possible to locate the site of injury, in two cases (Fig. 9). STIR sequences instead offered better visualisation of intramuscular oedema and perifascial fluid collection (Fig. $10)$, which were observable in all the cases assessed (Tab. I). We underline the fact that these signs were clearly observable in just a few sections. On the other hand, signal alterations in T1w sequences were minimal and offered little contribution for the diagnosis. No haematoma was detected in the first MRI acquisitions (Tab. I).

The muscle injury led to the local formation of intramuscular haematoma in 3 of 5 cases (Figs. 11, 12), as well as it was observed using ultrasound (see above). In such cases, haematoma-related signal al- 


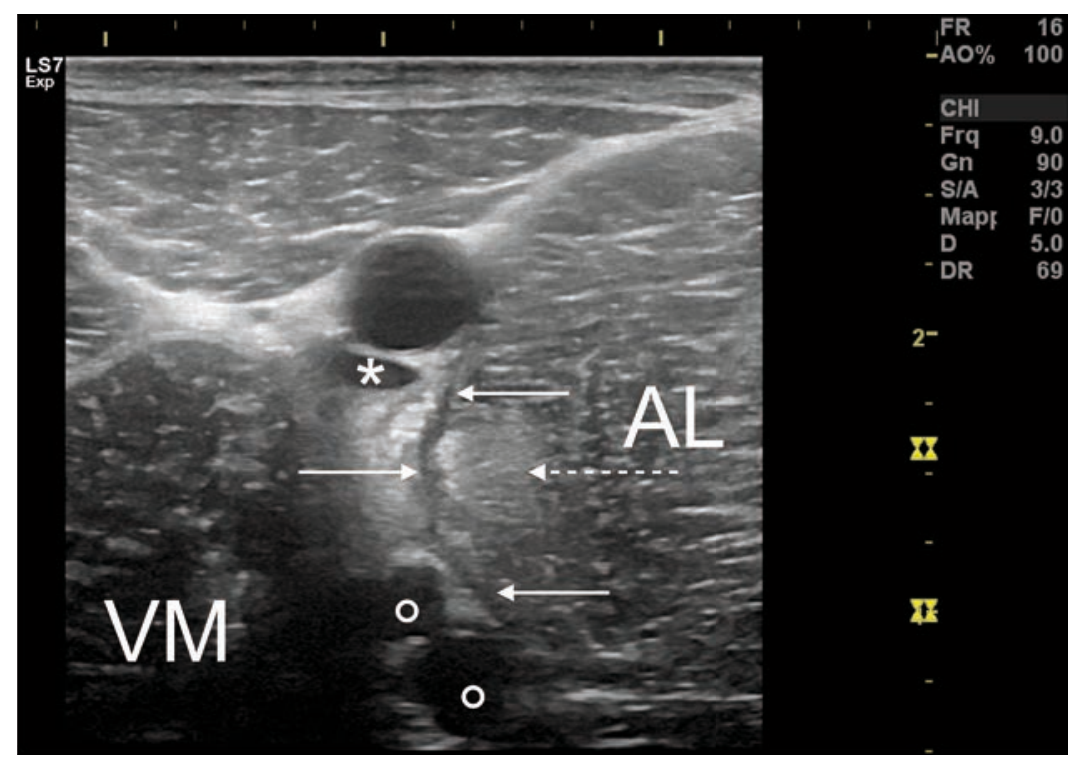

Figure 4. Cross-sectional ultrasound performed 48 hours post trauma showing the ultrasonographical features of the Perivascular Adductor Longus muscle injury in the acute phase. The eco-destructuration of the Adductor Longus ( $A L$ ) epimysium, which appears hypoechogenic, is indicated by the solid arrows and extends from the compressed femoral vein (asterisk) to the deep femoral vessels (circles). Oedema of AL within the muscle belly is visible in the vicinity of the lesion (dashed arrow). Due to this, the perimysium is not locally distinguishable from the muscle fibres. The hyperechoic region below the vessels and facing the Vastus Medialis (VM) is likely due to the posterior acoustic enhancement artifact induced by the femoral artery. Accordingly, at this site, the presence of interfascial liquid should not be confirmed using ultrasound.

terations were also visible in T1w sequences (Fig. 13). Interestingly, comparable findings were observed between the MRI performed at 3 weeks $/ 1$ month and at $2 / 3$ months post trauma. For this reason, longstanding oedema and haematoma have been considered the main long-term complications. In the perivascular Adductor Longus muscle injury, being classified as isolated "myofascial", there were no involvement of tendon structures, for which the formation of extensive fibrotic areas or perilesional calcifications did not occur in any of the cases.

\section{Injury mechanism}

Relevant information concerning the primary traumatic event is reported in Table II. Four footballers injured during an official or a friendly match. Three players experienced sudden and stabbing pain in their medial thigh region, which forced them to immediately suspend the sporting activity.

Three footballers reported that kicking was the traumatic gesture, while the other two players felt increasing soreness in the adductor region, without being able to identify the precise motor task that led to the injury. These two did not suspend the sporting activities due to the thigh pain; anyway, they sought medical attention and the first screening tests surprisingly revealed the muscle injury.

\section{Discussion}

The main purpose of this technical note was to raise awareness of the US and MRI features of the Perivascular Adductor Longus muscle injury. The major function of this article is to compensate for the lack of studies concerning the Perivascular Adductor Longus muscle injury, offering practical guidance on how to effectively investigate it via US and MRI. This article may be considered the point of reference for the imaging of this injury and for future related investigations.

\section{Ultrasound findings}

US was helpful as a first screening test and all the cases presented comparable findings in acute phase. These were consistent with those presented in the articles describing the US characteristics of muscle injuries $^{19-21}$. Despite this, misdiagnosis was a distinct possibility, particularly if the examination was carried out in the first seventy-two hours post-trauma; in fact the eco-structural abnormalities were of controversial analysis and interpretation in two of the five cases. In this sense, the Push-to-view tip has shown to have relevant practical value as it allowed to thoroughly evaluate the lateral peripheral portions of the $A L$ muscle belly, during the investigations; without applying this tip, the image quality decreased substantially, likely leading to misinterpretation. On the other hand, in the three cases in which an intramuscular haematoma formed, the associated US abnormalities were visible only at day 5 post-injury, or later, while, typically, their presence should be previously detectable ${ }^{19-21}$. Contrary to what is currently known about the evolution of the US features of the intra- 


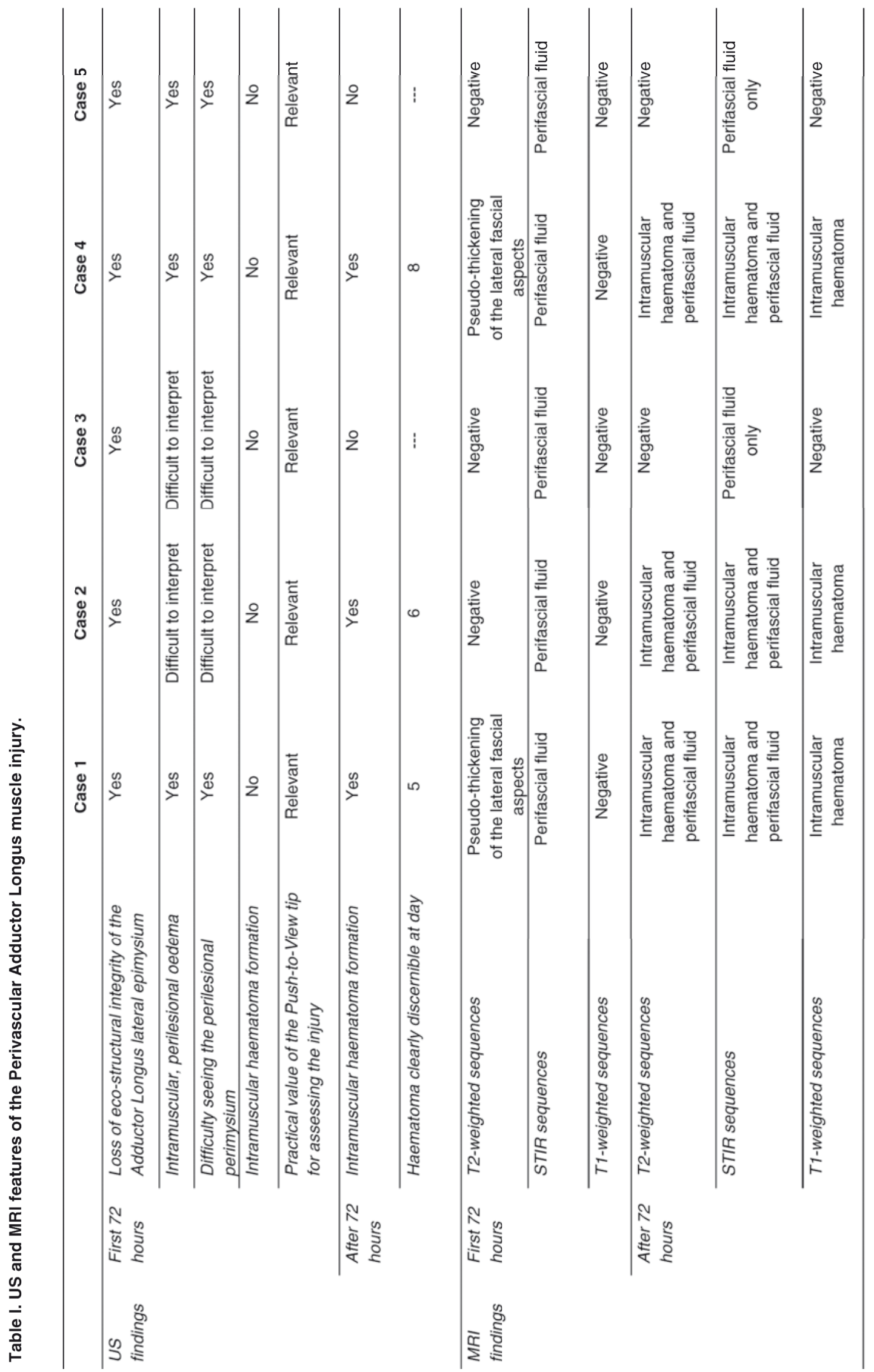




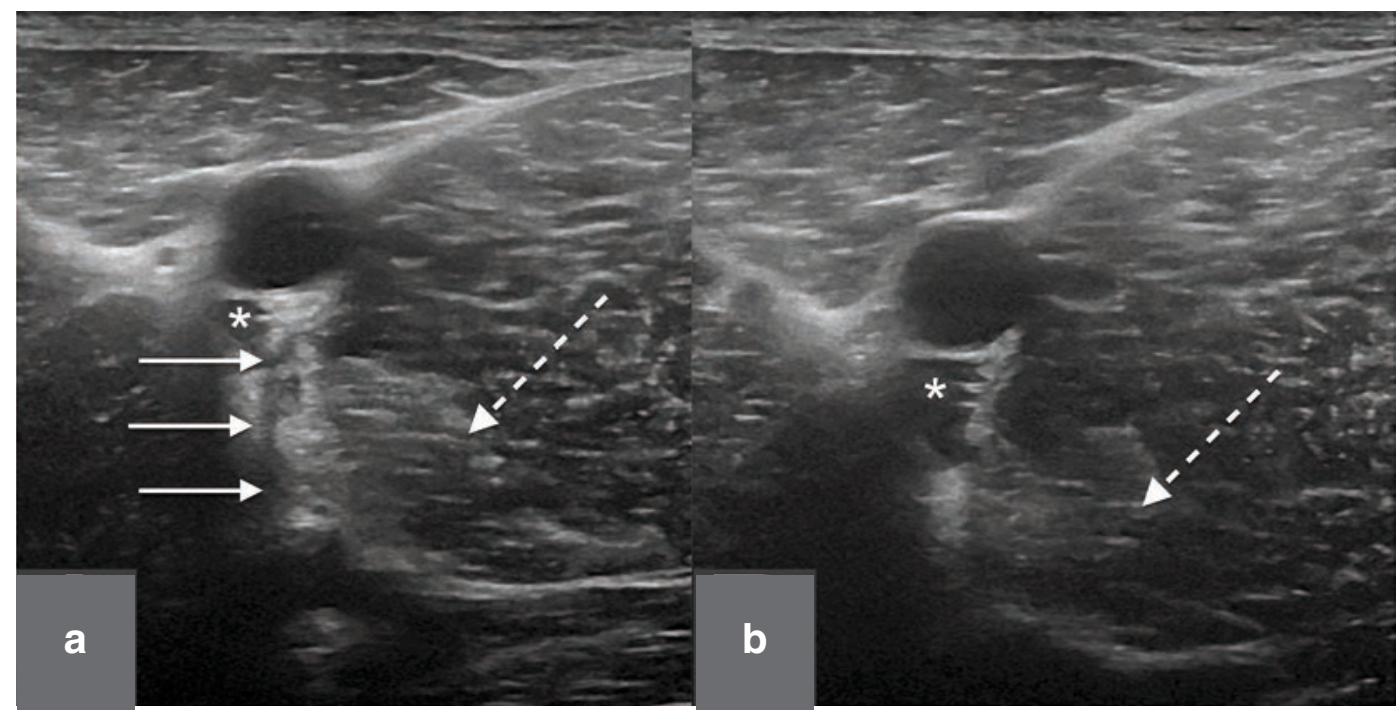

Figure 5 a, b. Practical value of the Push-to-View tip. Cross-sectional ultrasound images acquired in acute phase with (a) or without (b) the use of the tip. In (a), the eco-destructuration of the epimysium is well displayed (indicated by the solid arrows) as well as the intramuscular oedema (dashed arrow). In (b), the posterior acoustic enhancement artifact induced by the femoral vein (asterisk) significantly impairs the visualisation of the lesion; even the signs of the intramuscular oedema (dashed arrow), though present, are very weak in comparison. In this case, the possibility of misdiagnosis is certainly substantial.

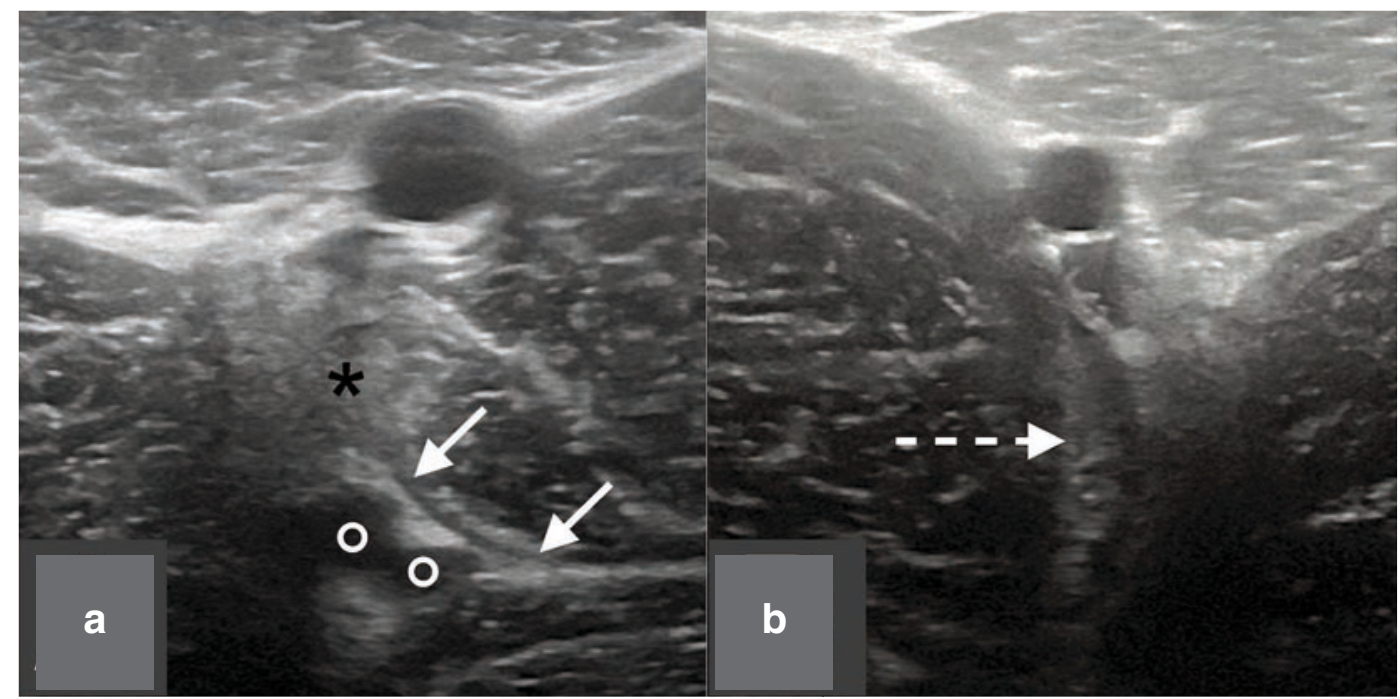

Figure 6 a, b. Ultrasound diagnostic difficulties in the analysis of the Perivascular Adductor Longus muscle injury, in acute phase. In (a), the compression of the femoral vein causes the formation of a wide echogenic area just under it (black asterisk), which prevents ideal visualisation of the injury. Despite this, the deepest part of the epymsium is still visible and appears hypoechogenic (solid arrows). The ultrasonographer must rule out that it is not from a horizontal blood vessel, originating from the deep femoral vessels (circles). In (b), while the eco-destructuration of the epymisium is quite evident, the intramuscular perilesional oedema (dotted arrow) is barely visible. There is also the possibility that this sign is mistaken for the posterior acoustic enhancement artifact caused by vessels.

muscular haematomas ${ }^{19}$, in no case we observed isoor hyperechoic areas within the muscle belly. This does not exclude that blood was present, at least, in the intra-fascial region. However, when clearly observable, the haematomas had clear hypoechogenic or anechoic appearance. According with the above, the final ultrasound diagnosis should not be estab- lished prior to one week post-trauma. Doing the opposite, entails the main risk of underestimating the actual severity of the injury.

\section{Magnetic Resonance Imaging findings}

Fat-suppressed fluid sensitive MRI techniques were the most useful to detect structural abnormalities in 


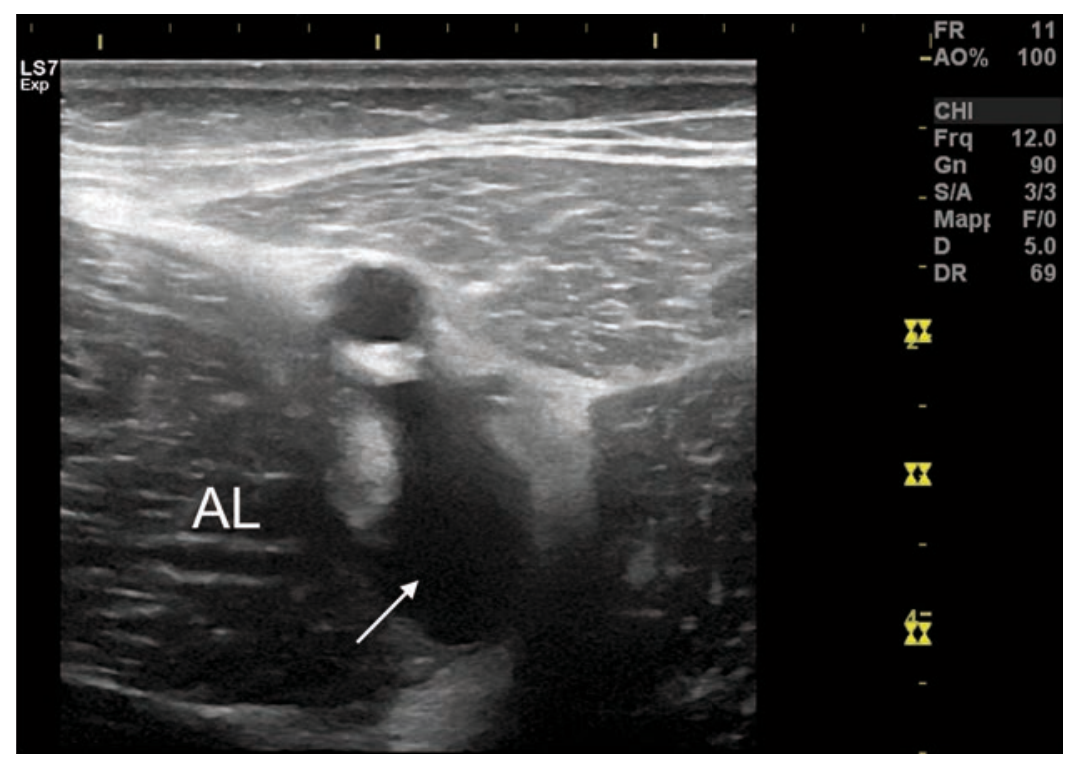

Figure 7. Cross-sectional ultrasound performed 7 days post trauma showing a high grade Perivascular Adductor Longus (AL) muscle injury. A wide anechoic region (solid arrow) is visible within the AL muscle belly. The sign is compatible with the presence of fluid, most likely blood, resulting from local lesion of the muscle fibres.

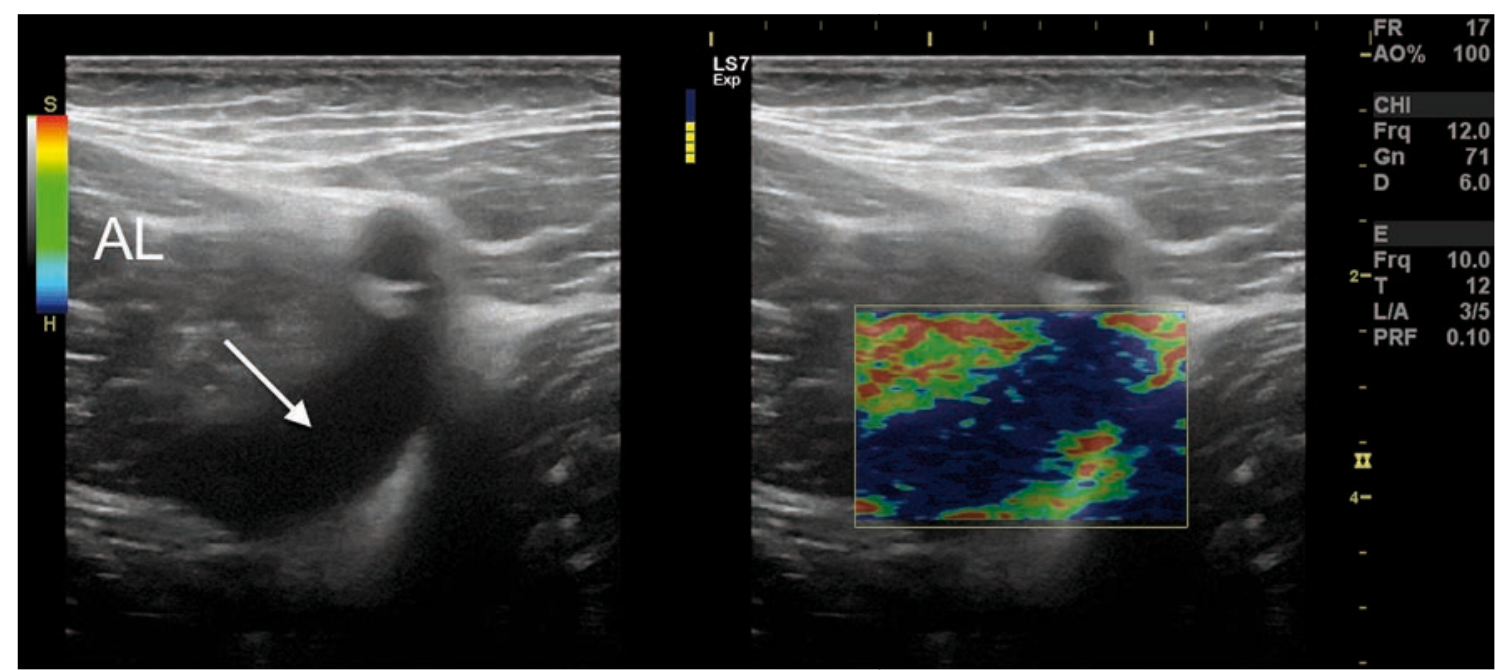

Figure 8. Elastosonography of an intramuscular haematoma within the lateral peripheral aspects of the Adductor Longus (AL). The fluid collection (full arrow) appears easily circumscribed as corresponding to the blue region, surrounded by green/red areas. Such collection is considered hard, i.e., with high mechanical resistance, potentially interpretable as a dense and not compressible liquid.

all phases of the healing process ${ }^{20}$. However, only the STIR sequences were positive in all the five cases, in the acute phase. Consequently, in case of suspected Perivascular Adductor Longus muscle injury, it is advisable to specifically review the sequences obtained using this technique. Despite this, haematomas-associated signal alterations were visible only in the MRI carried out after seventy-two hours, confirming the tendency to delayed formation of intramuscular haematomas of the Perivascular Adductor Longus muscle injury. It is worth noting that these signs were also visible at three months post-injury, even considering the T1-sequences, which typically have modest role in the assessment of muscle injuries $^{20}$. Long-standing haematoma (and related oedema) should be therefore considered the main complication of the Perivascular Adductor Longus muscle injury. Other structural abnormalities (large fibrotic areas and/or calcification) have not been observed in any of the cases. However, considering the low number of participants of this study, we did not definitely exclude that those may form. 

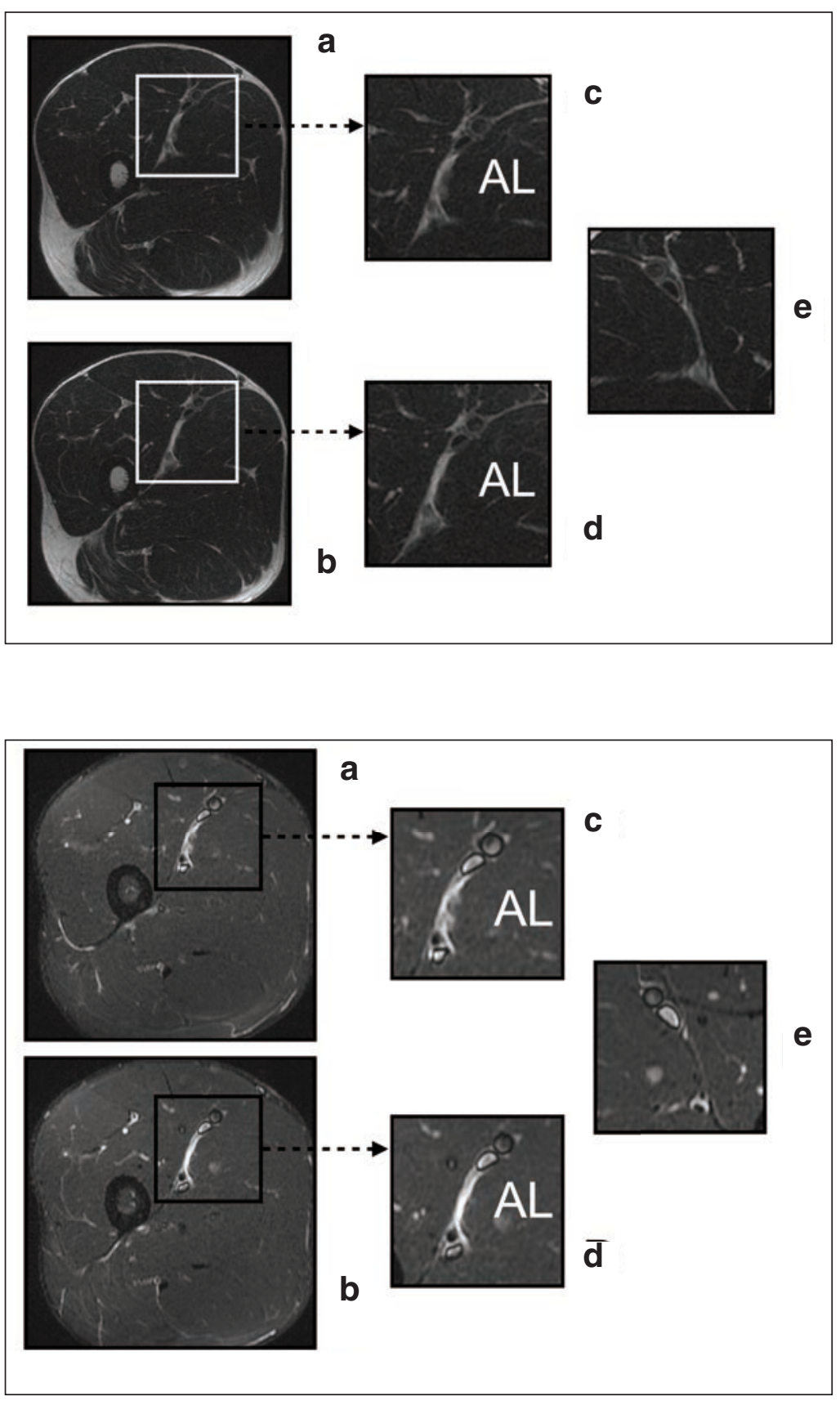

Figure 9 a-e. Fat-suppressed T2-weighted transversal consecutive sections showing the MRI features of Perivascular Adductor Longus $(A L)$ muscle injury, in the acute phase. In a and $c$ the intramuscular oedema, while in $b$ and $d$ the interfascial fluid, are observable. In c and $d$ the portions of interest of sections $\mathrm{a}$ and $\mathrm{b}$ (white squares) are magnified. In $\mathrm{c}$ it is worth noting how the oedematous area, corresponding to the region of increased intramuscular signal, does not extend to the Vastus Medialis, excluding lesions affecting this muscle. In $d$ the increase signal of the lateral epymisium mimics a pseudo-thickening of the fascia, which can be better assessed if compared with the same healthy portion of the contralateral limb (e). for a blood vessel.

\section{Injury mechanism and limitations of the study}

Kicking was reported to be the traumatic motor task in three cases. This gesture is already known to be implicated in the etiopathogenesis of $A L$ muscle injuries ${ }^{4}$. Interestingly, the other two players were not able to identify the gesture that led to the injury and, moreover, the onset of the symptoms was gradual. This suggests that clinical history may be misleading in case of Perivascular Adductor Longus muscle injury. In fact, when AL muscle injuries occur, the onset

Figure 10 a-e. Short tau inversion recovery transversal MRI consecutive sequences (corresponding to those presented in Figure 9). By comparing the sequences, sections $a$ and $b$ clearly show an increase in signal intensity in the lateral peripheral region of the Adductor Longus $(A L)$. The sections contained in the black squares are magnified and show the oedema (c) and the interfascial liquid (d) that specifically extend from the superficial femoral vessels to the deep ones (hence the "perivascular" prefix). This should be considered as the site to be reassessed in the sub-acute phase in order to evaluate the potential delayed intramuscular haematoma formation or interfascial fluid accumulation. The comparison with the contralateral limb (e) is always extremely useful as the signal alterations may be modest at this stage or present only in a few single sequences. In general, whenever is that the interfascial liquid is mistaken 


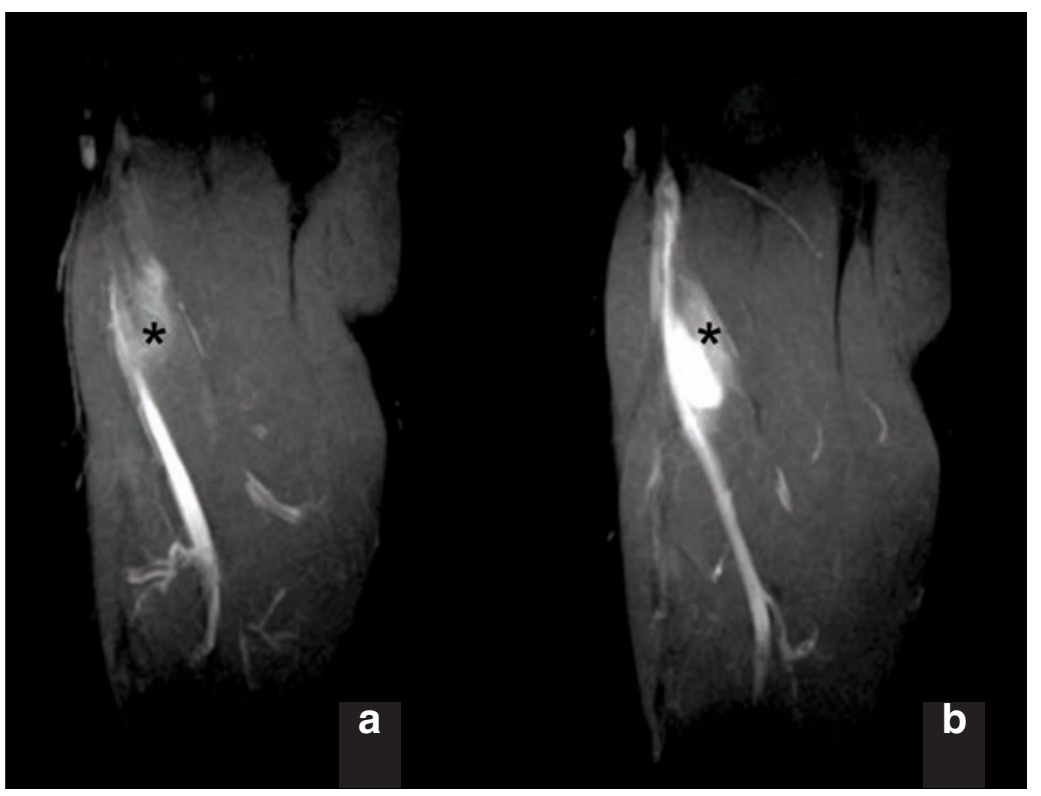

Figure $11 \mathrm{a}, \mathrm{b}$. Short tau inversion recovery sagittal MRI sequences acquired 30 days after trauma. In a the persistent and isolated intramuscular oedema (black asterisk), which extends for the entire section of the muscle, is visible. In b, the haematoma (large bright area) can easily be spotted, just slightly below the surface to the femoral artery and in relation with it.

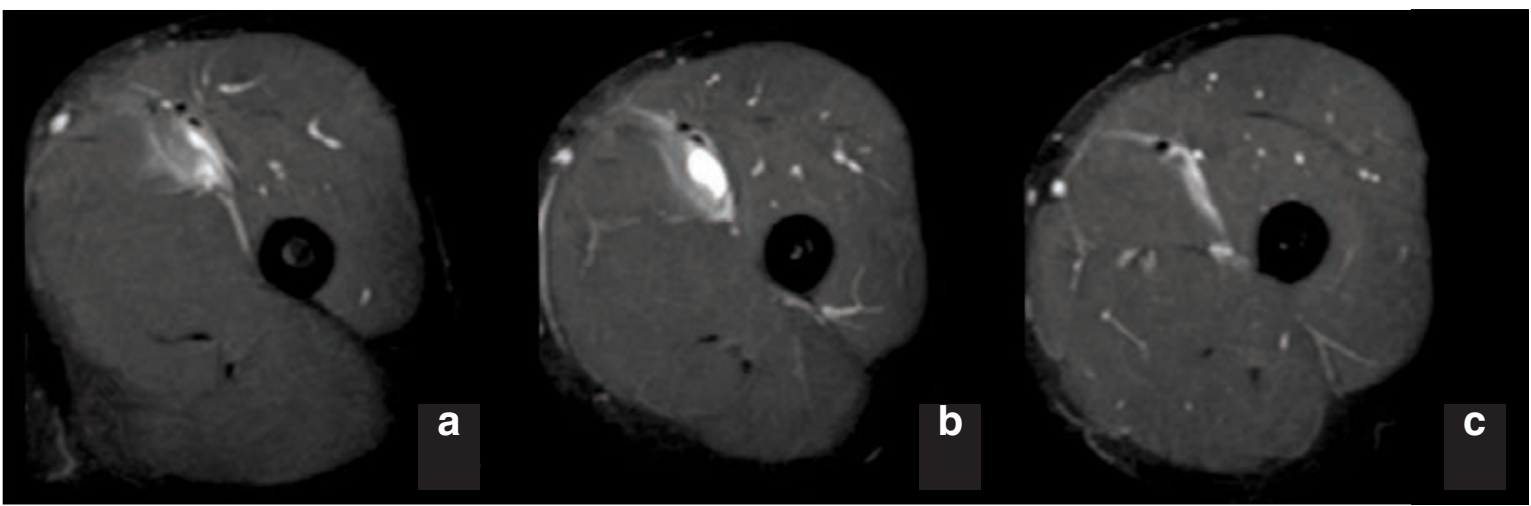

Figure 12 a-c. Short tau inversion recovery axial MRI sequences acquired at 30 days after trauma, from proximal to distal. The proximal section (a) shows the site as being likely to have been affected by the primary injury, with presence of interfascial effusion and intramuscular oedema, extending for about a third of the muscle belly. In b, fluid spillage, enclosed in the muscle belly, is clearly visible (bright area). It is situated just below the femoral vessels (two black points), possibly accumulated due to gravity. Just below the plausible haematoma, only a minimum increase of signal is observable (c) at epimysial level.

lack of this relevant information should be considered the main limitations of this article.

\section{Perivascular Adductor Longus muscle injury in the medical literature}

At the best of Authors' knowledge, there are only two more articles in literature that describe the clinical and/or the imaging characteristic of the Perivascular Adductor Longus muscle injury. In the report by Van De Kimmenade et al. $^{8}$, the case of a football player with long-standing (three months) thigh pain is presented. Kicking was the traumatic mechanism. Interestingly, the clinical tests were all substantially negative. On the contrary, the MRI images clearly showed a distal intramuscular haematoma. Our analysis of the transversal MRI sequences confirms it was a high grade Perivascular Adductor Longus muscle injury, since the signal alteration are specifically detectable between the $A L$ and the Vastus Medialis. Anyway, generic diagnosis of intramuscular $A L$ rupture was made. No proximal injuries are reported and no additional ultrasound images or follow-up MRI are presented in the paper.

On the other hand, in the article by Orlandi et al. ${ }^{10}$, an intramuscular Platelet-rich Plasma (PRP) injection procedure within the $A L$ muscle belly is presented. Revising the US-guided interventional techniques to treat sport-related muscle injuries was the main purpose of the paper. One of the US images provided shows an injury of the AL located just below the 


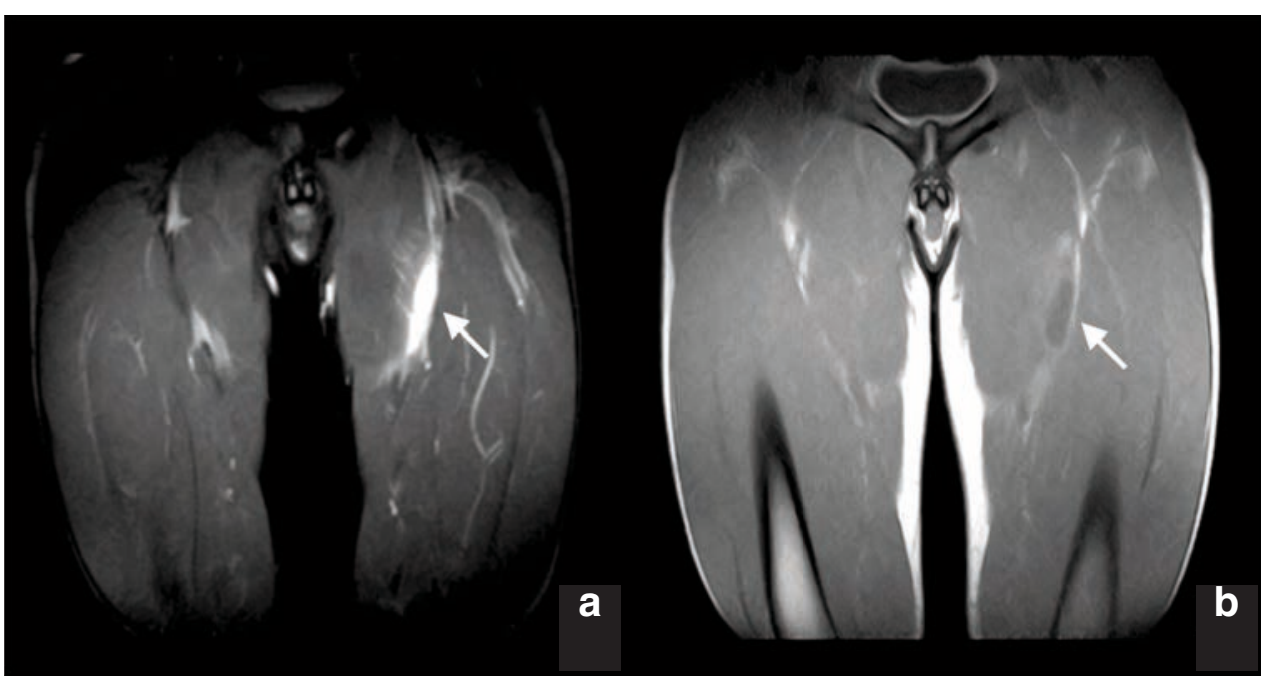

Figure $13 \mathrm{a}$, b. Coronal MRI sequences acquired 30 days post trauma. Large haematomas (white arrow) are recognizable both in STIR (a) and in T1-weighted (b) sequences, as hyperintense and hypointense areas, respectively.

Table II. Onset and mechanism of the Perivascular Adductor Longus muscle injury.

\begin{tabular}{|c|c|c|c|c|c|}
\hline & Case 1 & Case 2 & Case 3 & Case 4 & Case 5 \\
\hline Sporting activity & Match & Training & Match & Match & Match \\
\hline Onset & Sudden & Gradual & Sudden & Sudden & Gradual \\
\hline Motor Task & Kicking & Unknown & Kicking & Kicking & Unknown \\
\hline
\end{tabular}

femoral vessels. The US features of that injury are fully consistent with those of the Perivascular Adductor Longus muscle injury presented in our technical note. However, figure caption is somehow confounding as it indicates that the pubis, the proximal AL tendon and the femoral vessels would be visible in the same (transversal) section. This description creates an "anatomical paradox" since those structures cannot be this close, at any anatomical level. Despite this, the US images are overall of a high quality and show the evolution of the healing process of the injury (twenty days follow-up).

\section{Summary}

The Perivascular Adductor Longus muscle injury is still widely under-recognised and poorly and sparsely described in literature. Locating the presence of the injury and quantifying its extension is of vital importance to properly establish the prognosis and to rationally orientate the rehabilitation program, possibly optimising the healing process and reducing the risk of recurrence. Future studies are needed to identify the incidence of the Perivascular Adductor Longus muscle injury, which remains to this day unknown, among all the myotendinous injuries that affect the Adductor Longus, stating the return-to-play time depending on the degree of severity.

\section{Conflict of interest}

The Authors declare that they have no conflict of interest.

\section{References}

1. Dimitrakopoulou A, Schilders E, Bismil Q, Talbot JC, Kazakos K. An unusual case of enthesophyte formation following an adductor longus rupture in a high-level athlete. Knee Surg Sports Traumatol Arthrosc. 2010;18:691-693.

2. Thorborg K, Petersen J, Nielsen MB, Hölmich P. Clinical recovery of two hip adductor longus ruptures: a case-report of a soccer player. BMC Research Notes. 2013;6:205. 
3. Serner A, Tol JL, Jomaah N, Weir A, Whiteley R, Thorborg K, et al. Diagnosis of Acute Groin Injuries: a prospective study of 110 athletes. Am J Sports Med. 2015;43(8):1857-1864.

4. Charnock BL, Lewis CL, Garrett JR WE, Queen RM. Adductor longus mechanics during the maximal effort soccer kick. Sports Biomech. 2009;8(3):223-234.

5. Pesquer L, Reboul G, Silvestre A, Poussange N, Meyer P, Dallaudière $B$. Imaging of adductor-related groin pain. Diagn Interv Imaging. 2015;96:861-869.

6. Valent A, Frizziero A, Bressan S, Zanella E, Giannotti E, Masiero S. Insertional tendinopathy of the adductors and rectus abdominis in athletes: a review. MLTJ.2012;10(2):142148.

7. Brukner P, Connell D. 'Serious thigh muscle strains': beware the intramuscular tendon which plays an important role in difficult hamstring and quadriceps muscle strains. $\mathrm{Br} \mathrm{J}$ Sports Med. 2016;50:205-208.

8. Van De Kimmenade RJLL, Van Bergen CJA, Van Deurzen PJE, Verhagen RAW. A Rare Case of Adductor Longus Muscle Rupture. Case Rep Orthop. 2015;2015:840540.

9. Greditzer HG 4th, Nawabi D, Li AE, Jawetz ST. Distal rupture of the adductor longus in a skier. Clin Imaging. 2017;JanFeb:144-148.

10. Orlandi D, Corazza A, Arcidiacono A, Messina C, Serafini G, Scinfienza LM, et al. Ultrasound-guided procedures to treat sport-related muscle injuries. Br J Radiol. 2016;89:20150484.

11. Padulo J, Oliva F, Frizziero A, Maffulli N. Muscles, Ligaments and Tendons Journal - Basic principles and recommendations in clinical and field science research: 2016 update. MLTJ. 2016;6(1):1-5.

12. Peh WCG, Ng KH. Writing a technical note. Singapore Med J. 2010;51(2):101-104.

13. Strauss EJ, Campbell K, Bosco JA. Analysis of the Cross-Sectional Area of the Adductor Longus Tendon. A Descriptive
Anatomic Study. Am J Sports Med. 2007;35(6):996-999.

14. Davis JA, Stringer MD, Woodley SJ. New insights into the proximal tendons of adductor longus, adductor brevis and gracilis. Br J Sports Med. 2012;46:871-876.

15. Silvestri E, Muda A, Orlandi D. Adductor, Gracilis and Pectineus. In: Ultrasound Anatomy of Lower Limb Muscles: A Practical Guide. Spinger. 2015;75-89.

16. Taha AM. Ultrasound-Guided Obturator Nerve Block: A Proximal Interfascial Technique. Anesth Analg. 2012;114(1):236239.

17. Rha DW, Lee SH, Lee HJ, Choi YJ, Kim HJ, Lee SC. Ultrasound-Guided Injection of the Adductor Longus and Pectineus in a Cadaver Model. Pain Physician. 2015;18:e1111-1117.

18. Whittaker JL, Teyhen DS, Elliott JM, Cook K, Langevin HM, Dahl $\mathrm{HH}$, Stokes M. Rehabilitative ultrasound imaging: understanding the technology and its applications. J Orthop Sports Phys Ther. 2007;37(8):434-449.

19. Maffulli N, Oliva F, Frizziero A, et al. ISMuLT Guidelines for muscle injuries. MLTJ. 2014;24;3(4):241-249.

20. Crema MD, Yamada AF, Guermazi A, Roemer FW, Skaf AY Imaging techniques for muscle injury in sports medicine and clinical relevance. Curr Rev Musculoskeletal Med. 2015;8: 154-161.

21. Peetrons P. Ultrasound of muscles. Eur Radiol. 2002;12:3543.

22. Chan O, Del Buono A, Best TM, Maffulli N. Acute muscle strain injuries: a proposed new classification system. Knee Surg Sports Traumatol Arthrosc. 2012;20(11):2356-2362.

23. Pollock N, James SLJ, Lee JC, Chakraverty R. British athletics muscle injury classification: a new grading system. Br J Sports Med. 2014;48:1347-1351.

24. Serner A, Roemer FW, Hölmich P, et al. Reliability of MRI assessment of acute musculotendinous groin injuries in athletes. Eur Radiol. 2017;27(4):1486-1495. 